\title{
Comparison of Anti-Dengue and Anti-Zika IgG on a Plasmonic Gold Platform with Neutralization Testing
}

\author{
Alejandra Rojas, ${ }^{1}$ Muktha S. Natrajan, ${ }^{2}$ Jenna Weber, ${ }^{3}$ Fátima Cardozo, ${ }^{4}$ César Cantero, ${ }^{1}$ Jeyarama S. Ananta, ${ }^{5}$ Jessica Kost, ${ }^{5}$ \\ Meijie Tang, ${ }^{5}$ Sanny López, ${ }^{1}$ Cynthia Bernal, ${ }^{1}$ Yvalena Guillén, ${ }^{1}$ Laura Mendoza, ${ }^{4}$ Malvina Páez, ${ }^{4}$ Benjamin A. Pinsky, ${ }^{3,6}$ and \\ Jesse J. Waggoner $2,7 *$ \\ ${ }^{1}$ Departamento de Producción, Instituto de Investigaciones en Ciencias de la Salud, Universidad Nacional de Asunción, San Lorenzo, Paraguay; \\ ${ }^{2}$ Division of Infectious Diseases, Department of Medicine, Emory University, Atlanta, Georgia; ${ }^{3}$ Department of Pathology, Stanford University \\ School of Medicine, Palo Alto, California; ${ }^{4}$ Departamento de Salud Pública, Instituto de Investigaciones en Ciencias de la Salud, Universidad \\ Nacional de Asunción, San Lorenzo, Paraguay; ${ }^{5}$ Nirmidas Biotech Inc., Palo Alto, California; ${ }^{6}$ Division of Infectious Diseases and Geographic \\ Medicine, Department of Medicine, Stanford University School of Medicine, Stanford, California; ${ }^{7}$ Department of Global Health, Rollins School of \\ Public Health, Atlanta, Georgia
}

\begin{abstract}
Antibody cross-reactivity confounds testing for dengue virus (DENV) and Zika virus (ZIKV). We evaluated anti-DENV and anti-ZIKV IgG detection using a multiplex serological platform (the pGOLD assay, Nirmidas, Palo Alto, CA) in patients from the Asunción metropolitan area in Paraguay, which experiences annual DENV outbreaks but has reported few autochthonous ZIKV infections. Acute-phase sera were tested from 77 patients who presented with a suspected arboviral illness from January to May 2018. Samples were tested for DENV and ZIKV RNA by real-time reverse transcription-PCR, and for DENV nonstructural protein 1 with a lateral-flow immunochromatographic test. Forty-one patients (51.2\%) had acute dengue; no acute ZIKV infections were detected. Sixty-five patients (84.4\%) had anti-DENVneutralizing antibodies by focus reduction neutralization testing (FRNT $\left.{ }_{50}\right)$. Qualitative detection with the pGOLD assay demonstrated good agreement with $\mathrm{FRNT}_{50}(\mathrm{kappa}=0.74)$, and quantitative results were highly correlated between methods $(P<0.001)$. Only three patients had anti-ZIKV-neutralizing antibodies at titers of 1:55-1:80, and all three had corresponding DENV-neutralizing titers $>1: 4,000$. Hospitalized dengue cases had significantly higher anti-DENV IgG levels $(P<0.001)$. Anti-DENV IgG results from the pGOLD assay correlate well with FRNT, and quantitative results may inform patient risk stratification.
\end{abstract}

\section{INTRODUCTION}

During the 2015-2016 epidemic, Zika virus (ZIKV) cocirculated with dengue virus (DENV) in many regions of the Americas. ${ }^{1-3}$ This created difficulties in providing accurate etiologic diagnoses for symptomatic patients. Serologic methods are commonly used to detect a recent Flavivirus infection or past exposure. ${ }^{4,5}$ In addition, serologic testing can be used to quantify preinfection anti-DENV and anti-ZIKV antibody titers, which correlate with the risk of developing severe dengue during an acute infection. ${ }^{6-9}$ Differentiating antibody responses from individual Flavivirus species is complicated by the cross-reactivity of antibodies that develop after infections with these pathogens., ${ }^{5,10}$ For this reason, plaque reduction neutralization testing is considered as the reference serological method for human Flavivirus infections. However, neutralization testing, including modified methods such as focus reduction neutralization testing (FRNT), remains time- and resourceintensive, and antibody cross-reactions still occur. ${ }^{4,10-12}$

Our group previously described the development and initial evaluation of a duplex ZIKV-DENV ELISA on a plasmonic gold platform, the pGOLD assay (Nirmidas Biotech, Palo Alto, CA). ${ }^{13}$ This assay uses a microarray of viral antigens, spotted onto individual wells of a gold-plated biochip, to detect antibody isotypes in a secondary ELISA with fluorescently labeled antihuman antibodies. The pGOLD assay demonstrated sensitive detection of IgG against ZIKV and DENV, as well as the ability to accurately differentiate recent infections caused by these viruses. ${ }^{13}$ In contrast to FRNT, which requires days to complete, the *Address correspondence to Jesse J. Waggoner, Emory University
Department of Medicine, Division of Infectious Diseases, 1760
Haygood Dr. NE, Rm. E-132, Atlanta, GA 30322. E-mail: jjwaggo@ emory.edu
pGOLD protocol requires $\sim 2$ hours, and the platform allows for further multiplexing with additional viral antigens.

Paraguay experiences large annual DENV outbreaks with rates of infection that are among the highest in South America. ${ }^{14,15}$ Approximately $2 / 3$ of confirmed DENV infections occur in Asunción and the surrounding Central Department, although relatively little data on arboviral disease have been published from the country. ${ }^{14,16-21}$ In 2016, more than 200,000 suspected and probable dengue cases were reported in Paraguay, with only 571 suspected Zika cases. ${ }^{22-24}$ However, most of these cases could not be laboratoryconfirmed. In 2018, Paraguay again experienced a large dengue outbreak caused predominantly by DENV-1 without detection of ZIKV infections. ${ }^{14}$ In the current study, we sought to further evaluate the pGOLD assay in comparison with FRNT and study the detection of anti-DENV and anti-ZIKV antibodies in patient samples collected in Asunción during the 2018 DENV1 outbreak.

\section{MATERIALS AND METHODS}

Clinical samples and molecular testing. This protocol was reviewed and approved by the Scientific and Ethical Committees at the Instituto de Investigaciones en Ciencias de la Salud, Universidad Nacional de Asunción (IICS-UNA), and Emory University. Written and informed consent was obtained for all patients. Children older than 6 years provided assent.

Acute-phase sera were collected as part of a prospective study of arboviral infections in Asunción, which has been previously described. ${ }^{14}$ Patients were enrolled from January to May 2018. Nonstructural protein 1 (NS1) detection was performed on fresh serum using the immunochromatographic NS1 test in the Standard $Q$ Dengue Duo kit, performed according to the manufacturer recommendations (SD 
Biosensor, Suwon-si, South Korea). Sera were aliquoted and stored at $-80^{\circ} \mathrm{C}$ until use. Molecular testing was performed at IICS-UNA to 1) detect acute DENV, ZIKV, and chikungunya virus (CHIKV) infections, and 2) determine DENV serotype and quantify viral load using validated and published real-time reverse transcription ( $\mathrm{rRT}$ )-PCRs, as described. ${ }^{25-28}$ For the current study, sera were selected from 77 individuals who presented $\leq 8$ days after symptom onset and tested positive $(n=38)$ or negative $(n=39)$ for DENV by rRT-PCR.

Serological testing. Samples were tested with the pGOLDZika/dengue $\operatorname{lgG} / \operatorname{lgM}$ assay (the pGOLD assay), which was performed according to the manufacturer recommendations. The pGOLD assay includes the following antigens: inactivated viral lysate from DENV serotype 2 and ZIKV NS1. Each antigen in the pGOLD assay is spotted in triplicate. Results are expressed as a ratio of the average fluorescence of those three points divided by the mean fluorescence of a calibrator that is included on each pGOLD slide. IgG results were interpreted according to the manufacturer-recommended cutoff values. Anti-DENV IgG was positive at a ratio $\geq 0.076$. Anti-ZIKV IgG was positive at a ratio $\geq$ 0.28 in anti-DENV IgG-positive samples and $\geq 0.02$ in anti-DENV IgG-negative samples. Signal in the pGOLD assay was then normalized to the ratio used to define a positive result (e.g., 0.076 for anti-DENV lgG) such that a result $\geq 1$ was positive and a result $<1$ was negative for each antigen-lgG pair.

Sera were tested by FRNT for neutralizing antibodies against ZIKV, DENV-1, and DENV-2, as described. ${ }^{29,30}$ Focus reduction neutralization testing was interpreted as undetectable (titer $\leq 1: 15$ ) if $<50 \%$ neutralizing activity was observed in the first dilution relative to the control, or positive if $\geq 50 \%$ neutralization was observed in the first dilution. Samples with low-positive (titer $\leq 1: 200$ ) or negative $\mathrm{FRNT}_{50}$ results for DENV-1 and DENV-2 $(n=48)$ were tested for neutralizing antibodies against DENV-3 and DENV-4. This algorithm was used to decrease the FRNT test volume yet maintain the detection of DENV neutralizing antibodies. Viral strains used for neutralization testing were the following: ZIKV (PRVABC59 [KU501215.1]), DENV-1 (Hawaii), DENV-2 (New Guinea C), DENV-3 (Sleman/78), and DENV-4 (H241). ${ }^{29}$

Data from testing with in-house indirect ELISAs for antiDENV and anti-ZIKV IgG were available for 39/77 samples (50.6\%). ELISAs used whole viral antigen for both viruses, and were performed as described. ${ }^{31}$

Statistical analysis. Acute DENV infections were considered confirmed if DENV RNA and/or NS1 was detected in the acute-phase sample. The maximum neutralizing antibody titer for each patient was defined as the highest FRNT $_{50}$ titer detected against any of the tested serotypes. All statistical analyses were performed using GraphPad Prism version 8.0.1 (GraphPad, San Diego, CA). Categorical variables were compared by Fisher's exact test; continuous variables were compared by $t$-test or the Mann-Whitney test (viral load). Agreement between serological methods was evaluated by kappa statistic. Multivariate logistic regression was performed using the least-squares method, and model fits were compared by Akaike's information criterion corrected.

\section{RESULTS}

Clinical samples. Patients were enrolled in either an emergency care setting (Hospital Villa Elisa, $n=64$ [83.1\%]) or an outpatient setting (IICS-UNA, $n=13$ [16.9\%]), both located
TABLE 1

Distribution of patients in the study population according to demographic data and the detection of DENV RNA, DENV NS1, and neutralizing antibodies by focus reduction neutralization testing

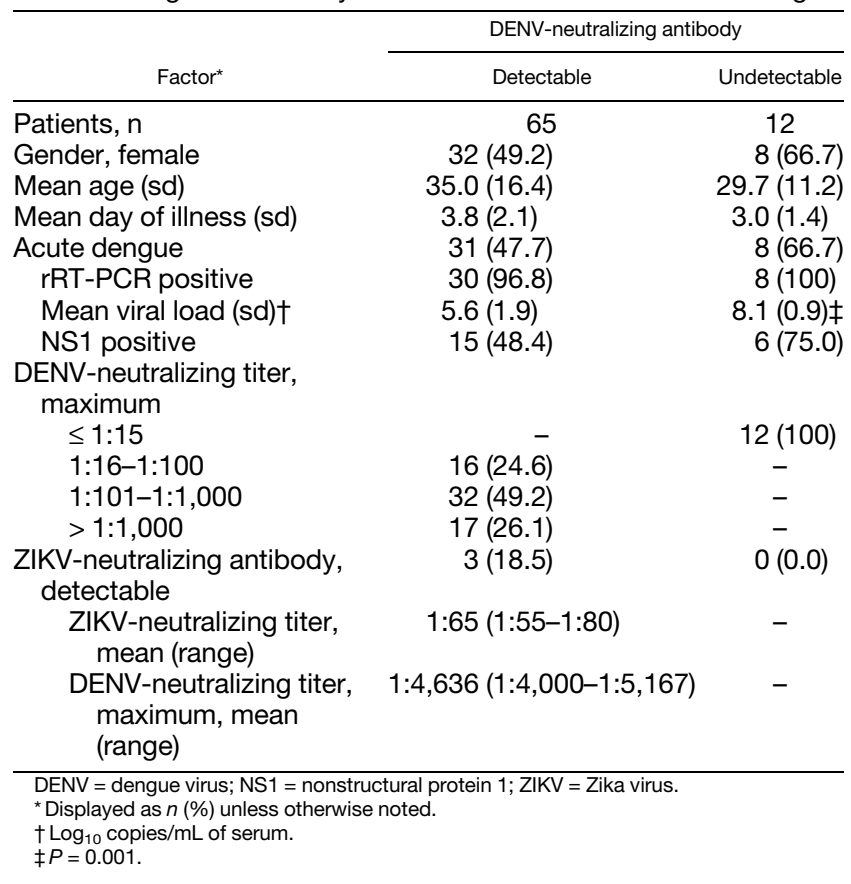

in metro Asunción. Demographic information and test results are shown in Table 1. Thirty-nine patients (50.6\%) had confirmed acute dengue based on the detection of DENV RNA and NS1 $(n=20)$, RNA alone $(n=18)$, or NS1 alone $(n=1)$. No acute ZIKV or CHIKV infections were detected.

Neutralization testing. Sixty-five of 77 patients (84.4\%) had anti-DENV-neutralizing antibodies (Table 1). Only three patients had detectable neutralizing antibodies against ZIKV and all at low titers (1:55-1:80; Table 1). Patients with ZIKVneutralizing antibodies presented 7-8 days after symptom onset and had anti-DENV-neutralizing titers of 1:4,000-1: 5,442 . Two of the three individuals tested positive for DENV-1 by $r$ RT-PCR.

IgG detection. Anti-DENV IgG was detected in 61/77 patients $(79.2 \%)$ at presentation using the pGOLD assay, which demonstrated good agreement with qualitative $\mathrm{FRNT}_{50}$ results for DENV (kappa statistic 0.74, 95\% Cl 0.54-0.94; Supplemental Table S1). The five samples with a positive FRNT $_{50}$ and negative pGOLD had low neutralization titers ( $n=$ 4 , maximum titer 1:42-1:80) or a monotypic neutralizing antibody response to one serotype ( $n=1$, anti-DENV-3 titer 1:385). There was a single positive pGOLD result in a patient without detectable neutralizing antibodies against DENV. Quantitative results in the PGOLD assay showed a significant correlation with the maximum $\mathrm{FRNT}_{50}$ titer (Figure $1 \mathrm{~A}, P<0.001$ ).

Thirteen samples tested positive for anti-ZIKV IgG in the pGOLD assay in the absence of ZIKV-neutralizing antibodies, yielding a specificity of $82.4 \%(61 / 74)$ compared with FRNT. All samples with detectable anti-ZIKV IgG in the pGOLD assay also had detectable anti-DENV IgG and anti-DENVneutralizing antibodies. For a given sample with detectable anti-ZIKV IgG in the pGOLD assay, the anti-DENV IgG signal, relative to the positive threshold, was always higher than the signal for anti-ZIKV IgG (Figure 1B). Anti-ZIKV IgG signal was 

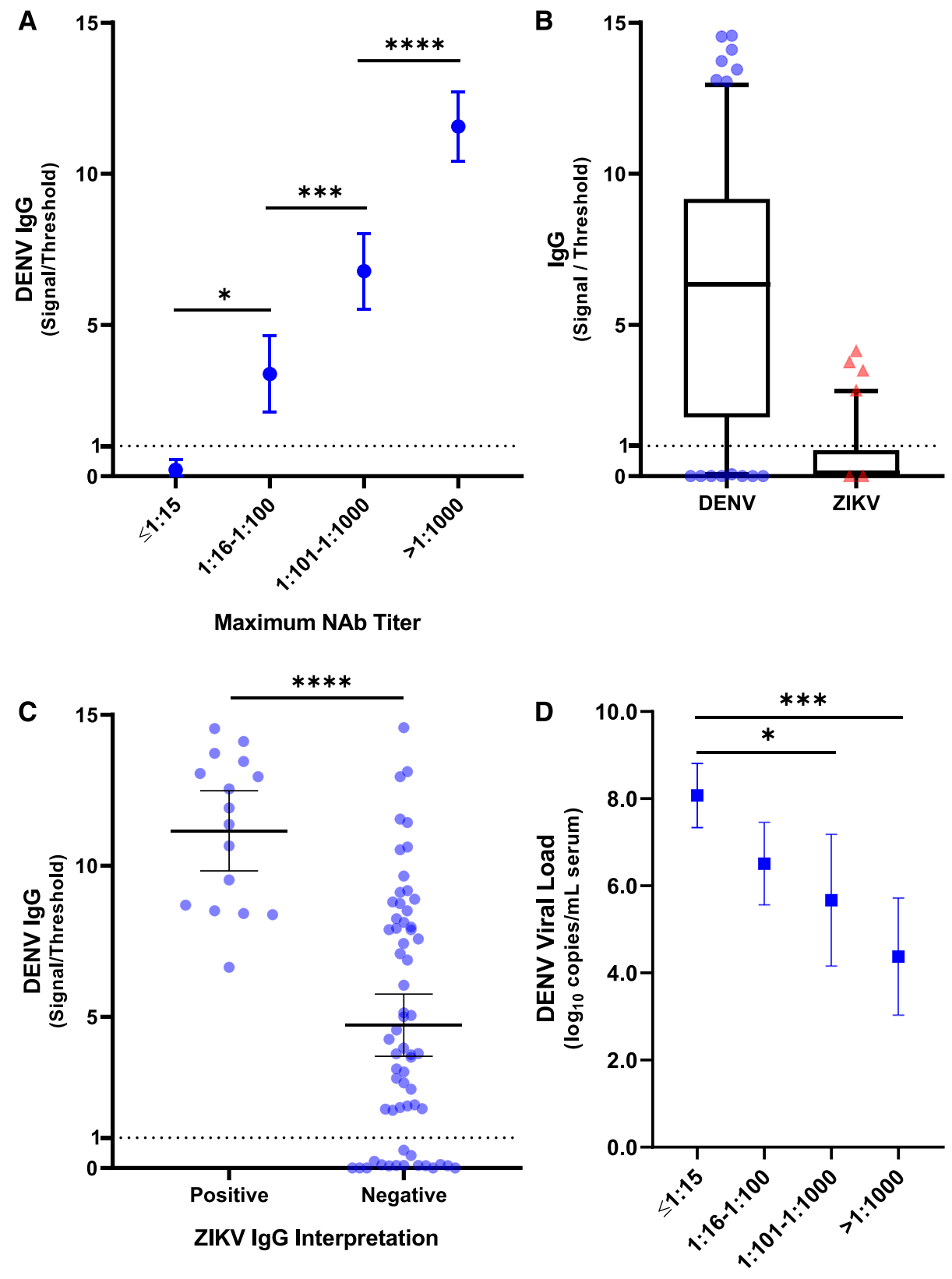

Maximum NAb Titer

FIGURE 1. Anti-DENV IgG detection in the pGOLD assay is associated with maximum anti-DENV titer by FRNT and serum viral load. Association between anti-DENV IgG titer in the pGOLD and the categorical, maximum anti-DENV titer measured by FRNT (maximum NAb titer). Mean values and 95\% Cl are displayed (A). Signal observed on the DENV antigen compared with the ZIKV antigen for individual samples (whiskers 10-90 percentile) (B). Comparison of anti-DENV IgG signal in samples that were positive and negative for anti-ZIKV IgG signal. Bars represent means and $95 \% \mathrm{Cl}$ (C). Linear correlation between DENV serum viral load and the maximum anti-DENV titer by FRNT (Maximum NAb Titer). Viral load means and $95 \%$ Cl are displayed (D); $P$-values are indicated on the graph: ${ }^{\star} P<0.05 ;{ }^{* \star \star} P<0.001 ;{ }^{* \star \star *} P<0.0001$. FRNT $=$ focus reduction neutralization testing; ZIKV $=Z$ ika virus. This figure appears in color at www.ajtmh.org.

only detected among samples with the highest titers of antiDENV IgG (Figure 1C and Supplemental Figure S1).

Finally, 39 samples were tested using in-house, indirect IgG ELISAs for DENV and ZIKV (Supplemental Table S2). With FRNT as the gold standard, the pGOLD assay was significantly more sensitive for anti-DENV IgG detection (35 versus 25 positives of $37 ; P=0.006$ ) and more specific with regard to the detection of signal on the ZIKV antigen (seven versus 21 positives of 38 samples that were negative for ZIKVneutralizing antibody; $P=0.002$ ).
Dengue virus viral load and hospitalization. There was a negative, linear correlation between DENV viral load and antiDENV titers (Figure 1D), and this effect remained significant after controlling for day of symptoms at presentation (Supplemental Table S3). Nine patients were hospitalized for dengue. Compared with outpatients, hospitalized cases had significantly higher levels of anti-DENV antibodies measured by either FRNT (Supplemental Figure S2) or the pGOLD assay ( $P<0.001$ for both methods), and the odds ratio for hospitalization among patients with anti-DENV IgG signal/threshold 
values $\geq 10$ in the pGOLD assay was 14.0 (95\% Cl: 2.4-61.8; $P=0.003)$. All six acute dengue cases (100\%) with anti-ZIKV IgG detected in the pGOLD assay had maximum DENV FRNT $_{50}$ titers $>1: 1,000$ and required hospitalization. Of the five other dengue cases with neutralization titers in this range but undetectable anti-ZIKV lgG, only one required hospitalization $(P=0.015)$.

\section{DISCUSSION}

This report builds on our previous results by evaluating the pGOLD assay against FRNT for anti-DENV and anti-ZIKV IgG in patients with an acute suspected arboviral illness. ${ }^{13}$ AntiDENV IgG detection in the pGOLD assay demonstrated good qualitative agreement with $\mathrm{FRNT}_{50}$. Quantitative results were highly correlated between these two methods and demonstrated a significant negative association with viral load, which has also been observed among dengue cases in a pediatric cohort study in Nicaragua. ${ }^{6}$ Using the pGOLD assay, we obtained results for anti-DENV and anti-ZIKV IgG in $\sim 2$ hours for 40 samples, similar to performance times for a single standard ELISA. However, the pGOLD assay proved significantly more sensitive and specific than in-house IgG ELISAs for DENV and ZIKV, demonstrating that this method warrants further study to potentially improve on available serologic methods.

Only three patients in our population had low-titer ZIKVneutralizing antibodies. Although potentially resulting from prior ZIKV infections, these results most likely represent cross-reactive binding antibodies that occurred in patients with secondary dengue. ${ }^{32}$ These data are consistent with the low numbers of Zika cases reported in Paraguay in 2015-2016..$^{22-24}$ By contrast, $84.4 \%$ of our population had DENV-neutralizing antibodies, demonstrating that high rates of ZIKV transmission by Aedes mosquito vectors could have occurred. ${ }^{23}$ The apparently low number of Zika cases in Paraguay may result from the fact that vector-borne ZIKV transmission is more temperature-dependent than DENV transmission. As such, subtle climatic differences between Asunción and neighboring regions may allow for high rates of DENV transmission in all areas yet limit ZIKV transmission in Paraguay. ${ }^{33}$

All six acute dengue cases with detectable anti-ZIKV IgG in the $p G O L D$ assay required hospitalization. These patients had high levels of anti-DENV IgG, consistent with secondary dengue, ${ }^{4,9,34}$ but the odds of hospitalization for these patients remained significantly elevated compared with other dengue cases with high levels of anti-DENV IgG. These anti-ZIKV antibodies were likely cross-reactive antibodies to DENV, but nonetheless, in the pGOLD assay, these antibodies target the NS1 antigen. The role of anti-NS1 antibodies in DENV infections remains unclear, ${ }^{35-37}$ but in a recent study, higher anti-NS1 antibody titers were observed among patients with dengue hemorrhagic fever. ${ }^{38}$ The detection of anti-NS1 antibodies along with quantitation of anti-DENV IgG may, therefore, provide data for risk stratification of dengue cases in the acute setting.

One limitation to this study was the use of acute-phase specimens. However, ZIKV-neutralizing antibodies from past infections should have been detectable even if seroprevalence estimates could not be calculated. Given the low level of anti-ZIKV antibodies in this population, the pGOLD assay should be further evaluated in a population with ongoing
ZIKV and DENV transmission. Finally, a limited FRNT workflow was used that may have affected the maximum antiDENV FRNT titers for a subset of samples. This would not have impacted the qualitative detection of anti-DENV or anti-ZIKV-neutralizing antibodies, and the effect on quantitative analysis is expected to be small, as only $7 / 48$ samples $(14.6 \%)$ had maximum titers against DENV-3 or DENV-4 when such testing was performed.

In conclusion, this study demonstrated the performance of the pGOLD assay for the detection and quantitation of antiDENV IgG and identified a potential role for its use in patient risk stratification in the acute setting.

Received November 10, 2020. Accepted for publication February 2, 2021.

Published online March 29, 2021.

Note: Supplemental tables and figures appear at www.ajtmh.org.

Acknowledgments: We thank the members of the study team based at the Instituto de Investigaciones en Ciencias de la Salud, Universidad Nacional de Asunción, and Hospital Villa Elisa in Paraguay for their dedication and excellent work, and we are grateful to the study participants and their families.

Financial support: Research was supported by NIH grants R21 Al131689 (B. A. P. and J. S. A.) and K08 Al110528 (J. J. W.). In addition, the development of this collaboration was supported by funding from the Consejo Nacional de Ciencia y Tecnología (CONACYT) in Paraguay (A. R.: PVCT16-66 and J. J. W.: PVCT17-65).

Disclosure: Three authors were employees of Nirmidas Biotech (JSA, $\mathrm{JK}$, and MT) and were involved in sample testing. All data were available to all authors for analysis.

Authors' addresses: Alejandra Rojas, César Cantero, Sanny López, Cynthia Bernal, and Yvalena Guillén, Departamento de Producción, Instituto de Investigaciones en Ciencias de la Salud, Universidad Nacional de Asunción, San Lorenzo, Paraguay, E-mails: arojass@ iics.una.py, cesarcantero24@gmail.com, sannylo2894@gmail.com, bernalcynthiaq@gmail.com, and ivalenaguillen@yahoo.com. Muktha S. Natrajan, Division of Bacterial Diseases, National Center for Immunization and Respiratory Diseases, CDC, Atlanta, GA, and Division of Infectious Diseases, Department of Medicine, Emory University, Atlanta, GA, E-mail: qdz9@cdc.gov. Jenna Weber, Department of Pathology, Stanford University School of Medicine, Palo Alto, CA, E-mail: jmicweber@gmail.com. Fátima Cardozo, Laura Mendoza, and Malvina Páez, Departamento de Salud Pública, Instituto de Investigaciones en Ciencias de la Salud, Universidad Nacional de Asunción, San Lorenzo, Paraguay, E-mails: fati.cardozo@hotmail.com, lauramendozatorres@gmail.com and paezmalvina@yahoo.es. Jeyarama S. Ananta, Jessica Kost, and Meijie Tang, Research and Development, Nirmidas Biotech Inc., Palo Alto, CA, E-mail: viskid@gmail.com, jessica.kost@nirmidas.com, and meijie.tang@nirmidas.com. Benjamin A. Pinsky, Division of Infectious Diseases and Geographic Medicine, Department of Medicine, Stanford University School of Medicine, Stanford, California, E-mail: bpinsky@stanford.edu. Jesse J. Waggoner, Department of Infectious Disease, Emory University, Atlanta, GA, E-mail: jesse.waggoner@ emoryhealthcare.org.

\section{REFERENCES}

1. Waggoner JJ et al., 2016. Viremia and clinical presentation in Nicaraguan patients infected with Zika virus, chikungunya virus, and dengue virus. Clin Infect Dis 63: 1584-1590.

2. Brasil $P$ et al., 2016. Zika virus outbreak in Rio de Janeiro, Brazil: clinical characterization, epidemiological and virological aspects. PLoS Negl Trop Dis 10: e0004636.

3. Silva MMO et al., 2019. Concomitant transmission of dengue, chikungunya, and Zika viruses in Brazil: clinical and epidemiological findings from surveillance for acute febrile illness. Clin Infect Dis 69: 1353-1359. 
4. World Health Organization, 2009. Dengue: Guidelines for Diagnosis, Treatment, Prevention and Control. Geneva, Switzerland: WHO Press.

5. Balmaseda A et al., 2018. Comparison of four serological methods and two reverse transcription-PCR assays for diagnosis and surveillance of Zika virus infection. J Clin Microbiol 56: e01785-e01817.

6. Waggoner JJ, Katzelnick LC, Burger-Calderon R, Gallini J, Moore RH, Kuan G, Balmaseda A, Pinsky BA, Harris E, 2020. Antibodydependent enhancement of severe disease is mediated by serum viral load in pediatric dengue virus infections. J Infect Dis 221: 1846-1854.

7. Katzelnick LC et al., 2020. Zika virus infection enhances future risk of severe dengue disease. Science 369: 1123-1128.

8. Salje $\mathrm{H}$ et al., 2018. Reconstruction of antibody dynamics and infection histories to evaluate dengue risk. Nature 557: 719-723.

9. Katzelnick LC, Gresh L, Halloran ME, Mercado JC, Kuan G, Gordon A, Balmaseda A, Harris E, 2017. Antibody-dependent enhancement of severe dengue disease in humans. Science 358: 929-932.

10. Lanciotti RS, Kosoy OL, Laven JJ, Velez JO, Lambert AJ, Johnson AJ, Stanfield SM, Duffy MR, 2008. Genetic and serologic properties of Zika virus associated with an epidemic, Yap State, Micronesia, 2007. Emerg Infect Dis 14: 1232-1239.

11. Roehrig JT, Hombach J, Barrett ADT, 2008. Guidelines for plaquereduction neutralization testing of human antibodies to dengue viruses. Viral Immunol 21: 123-132.

12. Rabe IB, Staples JE, Villanueva J, Hummel KB, Johnson JA, Rose L, Mts Hills S, Wasley A, Fischer M, Powers AM, 2016. Interim guidance for interpretation of Zika virus antibody test results. MMWR Morb Mortal Wkly Rep 65: 543-546.

13. Zhang $B$ et al., 2017. Diagnosis of Zika virus infection on a nanotechnology platform. Nat Med 23: 548-550.

14. Rojas A et al., 2019. Characterization of dengue cases among patients with an acute illness, Central Department, Paraguay. PeerJ 7: e7852.

15. Pan American Health Organization, 2020. Dengue. Available at: https:// www.paho.org/hq/index.php?option=com_topics\&view=article\&id= 1\&ltemid=40734\&lang=en. Accessed May 15, 2020.

16. Aquino JD, Tang WF, Ishii R, Ono T, Eshita $Y$, Aono H, Makino $Y$, 2008. Molecular epidemiology of dengue virus serotypes 2 and 3 in Paraguay during 2001-2006: the association of viral clade introductions with shifting serotype dominance. Virus Res 137: 266-270.

17. Aquino VH et al., 2006. Molecular epidemiology of dengue type 3 virus in Brazil and Paraguay, 2002-2004. Am J Trop Med Hyg 75: 710-715.

18. Cardozo F et al., 2018. Alphaviruses: serological evidence of human infection in Paraguay (2012-2013). Vector Borne Zoonotic Dis 75: 710-715.

19. Lovera D, Araya S, Mesquita MJ, Avalos C, Ledesma S, Arbo A, 2014. Prospective applicability study of the new dengue classification system for clinical management in children. Pediatr Infect Dis J 33: 933-935.

20. Lovera D, Martinez de Cuellar C, Araya S, Amarilla S, Gonzalez N, Aguiar C, Acuna J, Arbo A, 2016. Clinical characteristics and risk factors of dengue shock syndrome in children. Pediatr Infect Dis J 35: 1294-1299.

21. Rojas A, Aria L, de Guillen YA, Acosta ME, Infanzón B, Diaz V, López L, Meza T, Riveros O, 2016. Perfil clínico, hematológico y serológico en pacientes con sospecha de dengue del IICSUNA, 2009-2013. Mem Inst Investig Cienc Salud 14: 68-74.
22. Dirección General de Vigilancia de la Salud, Ministerio de Salud Pública y Bienestar Social, 2016. Boletín Epidemiológico: Semana Epidemiológica. Asunción, Paraguay: Ministerio de Salud Pública y Bienestar Social, 52.

23. Ministerio de Salud Publica y Bienestar Social, 2017. Boletín Epidemiológico República Del Paraguay. Asunción, Paraguay: Dirección General de Vigilancia de la Salud.

24. Vazquez C, de la Fuente AG, Villalba S, Torales J, Gamarra ML, Ortego MJ, Dunjo P, Chamorro G, Mendez J, Almiron M, 2019. Retrospective detection of Zika virus transmission in Paraguay January to December 2016. Weekly Epidem Rec 94: 161-168.

25. Waggoner JJ et al., 2013. Comparison of the FDA-approved CDC DENV-1-4 real-time reverse transcription-PCR with a laboratorydeveloped assay for dengue virus detection and serotyping. J Clin Microbiol 51: 3418-3420.

26. Waggoner JJ et al., 2013. Single-reaction, multiplex, real-time rtPCR for the detection, quantitation, and serotyping of dengue viruses. PLoS Negl Trop Dis 7: e2116.

27. Waggoner JJ, Gresh L, Mohamed-Hadley A, Ballesteros G, Davila MJ, Tellez Y, Sahoo MK, Balmaseda A, Harris E, Pinsky BA, 2016. Single-Reaction multiplex reverse transcription PCR for detection of Zika, chikungunya, and dengue viruses. Emerg Infect Dis 22: 1295-1297.

28. Cantero $C$ et al., 2020. Implementation of a multiplex rRT-PCR for Zika, chikungunya, and dengue viruses: improving arboviral detection in an endemic region. Am J Trop Med Hyg 102: 625-628.

29. Lai L et al.; Emory Zika Patient Study T, 2018. Innate, T-, and B-cell responses in acute human Zika patients. Clin Infect Dis 66: $1-10$.

30. Priyamvada $L$ et al., 2016. Human antibody responses after dengue virus infection are highly cross-reactive to Zika virus. Proc Natl Acad Sci U S A 113: 7852-7857.

31. El Sahly HM et al., 2019. Clinical, virologic, and immunologic characteristics of Zika virus infection in a cohort of US patients: prolonged RNA detection in whole blood. Open Forum Infect Dis 6: ofy352.

32. Collins MH, McGowan E, Jadi R, Young E, Lopez CA, Baric RS, Lazear HM, de Silva AM, 2017. Lack of durable crossneutralizing antibodies against Zika virus from dengue virus infection. Emerg Infect Dis 23: 773-781.

33. Tesla B, Demakovsky LR, Mordecai EA, Ryan SJ, Bonds MH, Ngonghala CN, Brindley MA, Murdock CC, 2018. Temperature drives Zika virus transmission: evidence from empirical and mathematical models. Proc Biol Sci 285: 20180795

34. Guzman MG, Harris E, 2015. Dengue. Lancet 385: 453-465.

35. Glasner DR, Puerta-Guardo H, Beatty PR, Harris E, 2018. The good, the bad, and the shocking: the multiple roles of dengue virus nonstructural protein 1 in protection and pathogenesis. Annu Rev Virol 5: 227-253.

36. Shu PY, Chen LK, Chang SF, Yueh YY, Chow L, Chien LJ, Chin C, Lin TH, Huang JH, 2000. Dengue NS1-specific antibody responses: isotype distribution and serotyping in patients with dengue fever and dengue hemorrhagic fever. J Med Virol 62: 224-232.

37. Nascimento EJM, Huleatt JW, Cordeiro MT, Castanha PMS, George JK, Grebe E, Welte A, Brown M, Burke DS, Marques ETA, 2018. Development of antibody biomarkers of long term and recent dengue virus infections. J Virol Methods 257: 62-68.

38. Jayathilaka D et al., 2018. Role of NS1 antibodies in the pathogenesis of acute secondary dengue infection. Nat Commun 9: 5242. 